\title{
Simulation study on hydrogen venting and diffusion from the safety valve of liquid hydrogen tank turning on to its turning off
}

\author{
Cunyan Cui ${ }^{\mathrm{a}}$, Yuan Li, Donglei Fan, Yachong Wang and Ningyuan Liu \\ Department of Space Equipment, Equipment Academy, Beijing, Huairou Zone, China
}

\begin{abstract}
Simulation study on hydrogen venting from liquid hydrogen tank of a certain rocket and diffusion form the safety valve of liquid hydrogen tank turning on to its turning off is carried out. Geometry model of safety valve of liquid hydrogen tank is established. And hydrogen venting process is studied when the pressure in the tank is as high as the limited pressure which is $0.35 \mathrm{MPa}$. Hazard zone produced by hydrogen cloud is analyzed and it is valuable for establishing some predicted method to meet possible emergency.
\end{abstract}

Keywords: hydrogen venting; rocket tank; diffusion; safety valve.

\section{Introduction}

Hydrogen vent-pipe will fall off when liquid hydrogen filling of a certain rocket tank is over. Then hydrogen vent pipe will fall off and hydrogen exhausting process is ceased. The tank will absorb quantity of heat from ambience for the temperature of liquid hydrogen in it is only about 20K. The heat will make liquid hydrogen evaporate, and the pressure in the tank will rise as a result. Safety valve will turn on automatically when the tank pressure is up to $0.35 \mathrm{MPa}$ in order to exhaust the hydrogen to ensure the tank is safe. The safety valve will turn off automatically when the pressure is about $0.3 \mathrm{MPa}$ for the safe pressure of the tank is from $0.3 \mathrm{MPa}$ to $0.35 \mathrm{MPa}$. The exhausted hydrogen is a high dangerous source for it is easy to be ignited when its density is $4 \%-7 \%$. So it is very important to study diffusion process of hydrogen.

In 1988, Shebeko et al[1] carried out some study on hydrogen diffusion in sealed space such as pipe, underground park, etc. the results showed that the jet kinetic energy is the main factor to influence the hydrogen diffusion at initial stage, and buoyancy became the main factor at the falling time. In 2000, Michael R. Swain[2] established a method to named HRAM to evaluated the hazard after the hydrogen leaked. And a kind of CFD software named FLUENT is used to simulated hydrogen leaking and diffusion process. And this method is adopted in this paper.

\section{Geometrical model used in simulation}

Geometrical model of safety valve of liquid hydrogen tank is established as shown in Fig. 1. Three parts named A, B, C constitute the whole geometry model. The length of the three sides of Part A is

a Corresponding author : ccy6655@126.com

(c) 2016. The authors - Published by Atlantis Press 
$x=2 \mathrm{~m}, y=1.5 \mathrm{~m}, z=1 \mathrm{~m}$, respectively. Part B is a column of which the diameter is $60 \mathrm{~mm}$ and its high is $100 \mathrm{~mm}$. The length of the three sides of Part $C$ is $x=20 m, y=8 m, z=5 m$. The gravity directs negative direction of the axis of $y$. Part $A$ is full of hydrogen gas with low temperature, part $C$ is in normal temperature and atmospheric pressure, and part $B$ is vent hole of which the coordinate is $(0.1,0,0)$.

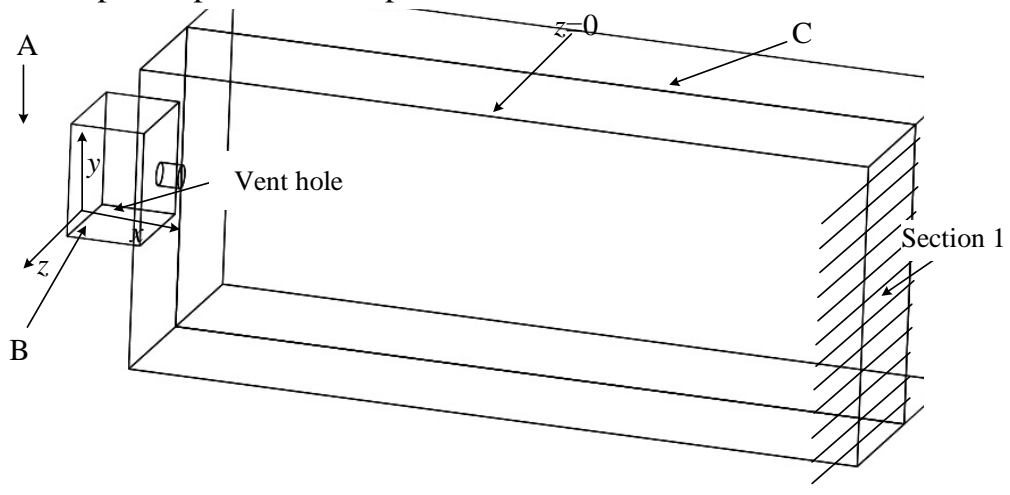

Figure 1. Three dimensional geometry model of hydrogen vent hole

\section{Discussions}

Monitoring point E(-1,0,0)is selected in order to observe the pressure variation after safety valve opened. Curve of variation of the pressure with time is shown in Fig. 2 in which coordination of $(0.43,0.30)$ express as the tank pressure falls from $0.35 \mathrm{MPa}$ to $0.3 \mathrm{MPa}$ within $0.43 \mathrm{~s}$. The safety valve will turns off automatically when the pressure is about $0.3 \mathrm{MPa}$.

Two sections at which $z=0$ and $y=0$ are selected to observe the hydrogen diffusion when the time is $0.1 \mathrm{~s}, 0.2 \mathrm{~s}, 0.3 \mathrm{~s}$, and $0.4 \mathrm{~s}$. The hydrogen concentration contour lines are shown in Fig. 3 and Fig. 4. We make $\mathrm{m}$ represents horizontal diffusion distance of the hydrogen, and $\mathrm{l}$ is uprightness distance, and $\mathrm{k}$ is the transverse size of the hydrogen cloud.

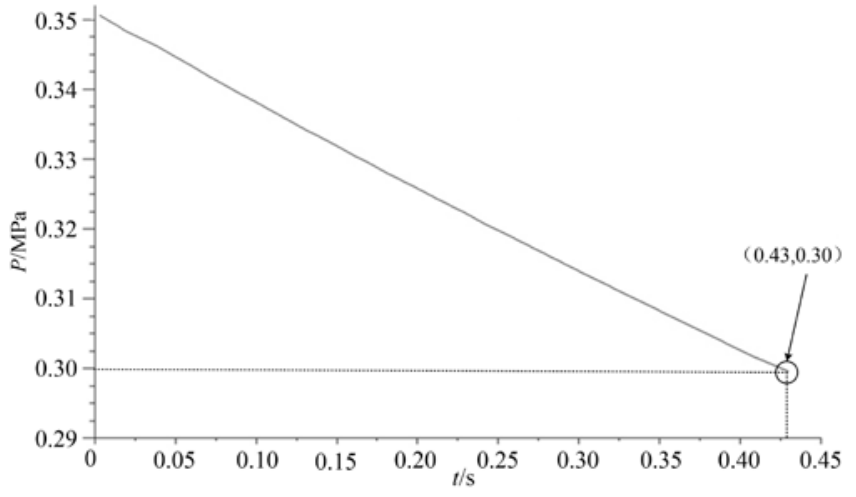

Figure 2. The variation curve of tank pressure from the safety valve turning on to it turning off

Hydrogen concentration contour lines at different time at the section of $z=0$ are shown in Fig. 3. The line at outermost layer means that the hydrogen concentration is $4 \% . m=1.5 \mathrm{~m}$ and $l=1.03 \mathrm{~m}$ respectively at $t=0.1 \mathrm{~s}$. The cloud enlarges with the hydrogen vents more, and $m=4.8 \mathrm{~m}$ while $l=3.0 \mathrm{~m}$ at $t=0.4 \mathrm{~s}$. 


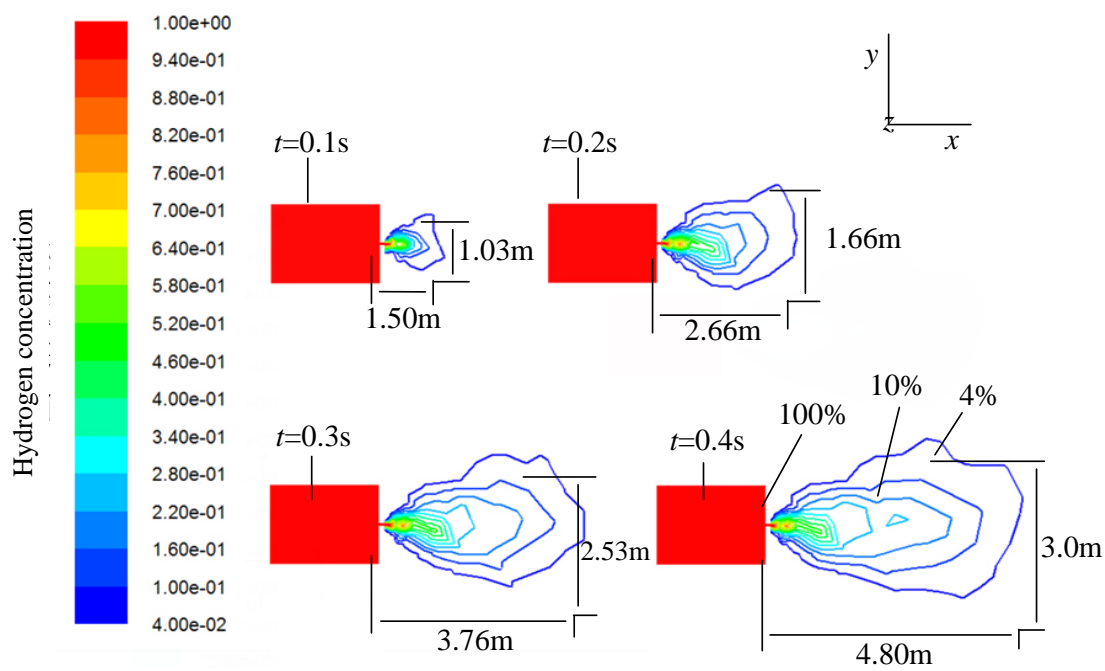

Figure 3. Hydrogen concentration contour lines at different time at the section of $z=0$

Hydrogen concentration contour lines at different time at the section of $y=0$ are shown in Fig. 4 from the safety valve turning on to it turning off. The value of $k$ is $1.0 \mathrm{~m}$ at $t=0.1 \mathrm{~s}$, and $k=3.33 \mathrm{~m}$ at $t=0.4 \mathrm{~s}$. The values of $\mathrm{m}, l$ and $k$ are shown in Fig. 5 when the time is from $0 \mathrm{~s}$ to $0.4 \mathrm{~s}$. It is obvious that the value of $\mathrm{m}$ is proportional to $\mathrm{t}$ when $\mathrm{t}=0-0.4 \mathrm{~s}$. The hydrogen speed is so large that the buoyant force has little effect on the values of $l$ and $k$, and so has the ambient environment. The section of the combustible is almost symmetrical during diffusion process.

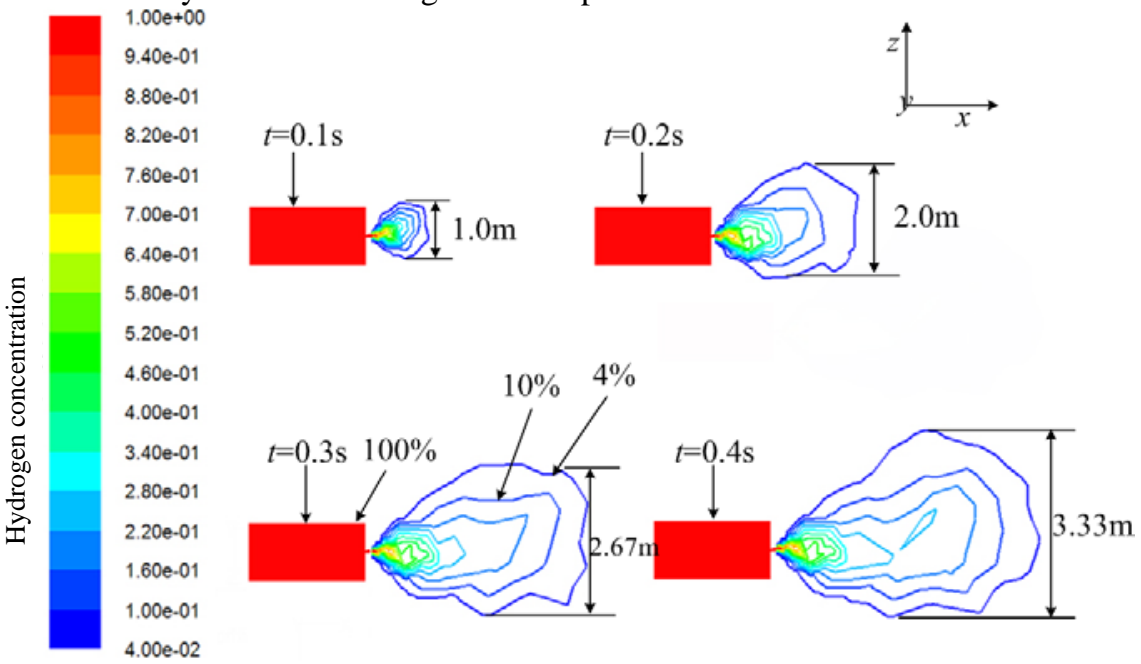

Figure 4. Hydrogen concentration contour lines at different time at the section of $y=0$ 


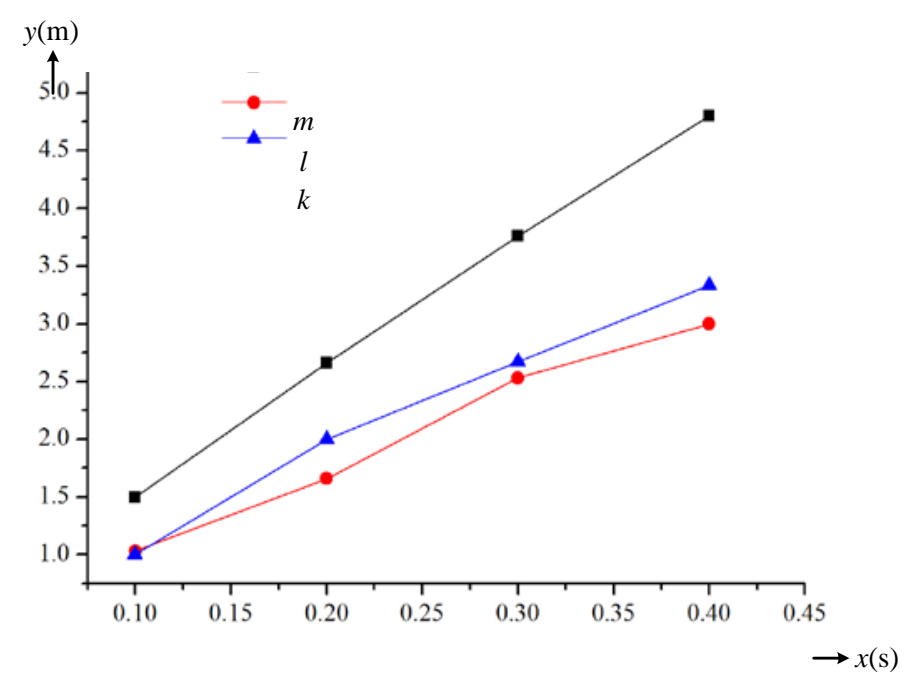

Figure 5. The variation of the values of $\mathrm{m}, \mathrm{l}$ and $\mathrm{k}$ from the safety valve turning on to it turning off

\section{Summary}

Liquid hydrogen tank of a certain rocket is in danger when the pressure in the tank is high enough. One of the most dangerous results is leakage. Dangerous regions are figured out in this paper with varied leakage conditions. And the study results are useful for preventing people and some key equipment from being harming.

\section{References}

1. A. Dewan, R. Angal, K. A. Subramanian. Computational Study of Turbulent Hydrogen Dispersion Hazards in a Closed Space[J]. The IUP Journal of Mechanical Engineering, Vol.5(012), pp. 28-42.

2. M. R. Swain, S. Eric. Advances in hydrogen energy[M]. Kluwer Academic/Plenum Publishers, Vol.(2000), pp.167-173. 